(Aus dem physikalischen Institut der Universität Rostock.)

\title{
Ueber aplanatische Brechung und Spiegelung in Oberflächen zweiter Ordnung und die Horn- hautrefraction.
}

Von

\section{Ludwion Nathiessen.}

Es ist seit langer Zeit bekannt, dass homocentrische Strahlenbündel in krummen Oberflächen im Allgemeinen astigmatisch gebrochen und gespiegelt werden. Doch ist auch längst bekannt, dass es krumme Oberflächen gibt, in denen Strahlenbündel homocentrisch gebrochen oder gespiegelt werden, wesshalb man sie aplanatische Flächen nennt. Solche sind gewisse Rotationsflächen vierter Ordnung, z. B. die Cartesischen Ovale. Cartesius suchte sie auf die Construction von Glaslinsen anzuwenden. Es ist nun freilich von grösserem praktischem Interesse, zu wissen, dass für gewisse Strablendurchgänge resp. Reflexionen auch sämmtliche Rotationsflächen zweiter Ordnung oder Kegelschnitte, diese in ihrer ganzen Ausdehnung aplanatische Flächen sind, wie ich bereits in früheren Abhandlungen für specielle Fälle, namentlich für die ellipsoidische Hornhaut des menschlichen Auges, gezeigt habe. Es sollen hier folgende Theoreme bewiesen werden.

1. Theorem: Wenn bei einer beliebigen Rotationsfläche zweiter Ordnung ein gespiegeltes, unendlich dünnes Strahlenbündel ent weder direct oder in seiner Verlängerung durch einen Focus geht, so ist die Brechung aplanatisch.

2. Theorem: Wenn bei einer beliebigen Rotationsfläche zweiter Ordnung ein gebrochenes Strahlenbündel eines sehrentfernten leuchtenden Punktes durch einen Focus geht, so ist die Brechung aplanatisch.

3. Theorem: Wenn die vorerwähnten Strahlenbündel nach ihrer Spiegelung oder Brechung in einer ebenen 
Curve oder Rotationsfäche durch einen festen Punkt gehen, so ist die Curve ein Kegelschnitt oder die Rotationsfläche eine Fläche zweiter Ordnung und der feste Punktein Focus.

Um diese Sätze zu beweisen, leiten wir zunächst die Bedingungsgleichungen $a b$, unter welchen Aplanatismus stattfindet. Wir gehen dabei aus von den bekannten Reusch'schen Formeln, welche einen speciellen Fall der allgemeinen Formeln von Carl Neumann für beliebige krumme Flächen bilden, und welche gültig sind für den Fall, wo der leuchtende Punkt im Hauptnormalschnitte des Finfallspunktes liegt. Es ist dann

$$
\begin{aligned}
& \text { I. } \frac{-\varrho_{1} \sin e_{1}}{\sin \left(e_{2}-e_{1}\right)} \cdot \frac{\cos e_{2}{ }^{2}}{x_{0}}+\frac{\varrho_{1} \sin e_{2}}{\sin \left(e_{2}-e_{1}\right)} \cdot \frac{\cos e_{1}{ }^{2}}{x_{1}}=1, \\
& \text { II. } \frac{-\varrho_{2} \sin e_{1}}{\sin \left(e_{2}-e_{1}\right)} \cdot \frac{1}{x_{0}}+\frac{\varrho_{2} \sin e_{2}}{\sin \left(e_{2}-e_{1}\right)} \cdot \frac{1}{x_{2}}=1 .
\end{aligned}
$$

Hierin bedeuten $e_{2}$ und $e_{1}$ den Einfalls- und Brechungswinkel, $\varrho_{1}$ und $\varrho_{2}$ die Krümmungsradien des Haupt- und Nebennormalschnittes der Fläche, $x_{0}$ die Abscisse des leuchtenden Punktes auf dem einfallenden Strahle, $x_{1}$ und $x_{2}$ die Abscissen der Bilder $B_{1}$ und $B_{2}$ auf dem gebrochenen oder gespiegelten Achsenstrahle des Lichtbündels. Lösen wir die Gleichungen nach $x_{1}$ und $x_{2}$ auf und setzen für den Aplanatismus $x_{1}=x_{2}$, so erhalten wir seine Bedingungsgleichung

III a. $x_{0}\left(\varrho_{2}-\varrho_{1} \cos e_{1}^{2}\right)=\sin e_{1} \sin \left(e_{2}+e_{1}\right) \varrho_{1} \varrho_{2}$,

und für die Bilddistanz

III b. $x_{2}\left(\varrho_{2}-\varrho_{1} \cos e_{2}^{2}\right)=\sin e_{2} \sin \left(e_{2}+e_{1}\right) \varrho_{1} \varrho_{2}$.

Hieraus ergibt sich nun, dass für Spiegelung d. h. $e_{1}=-e_{2}$, die Bedingungsgleichung III a lautet für beliebige $x_{0}$

$$
\varrho_{2}=\varrho_{1} \cos e_{1}{ }^{2}
$$

und da für eine Rotationsfläche $\varrho_{2}=N$ (Normale des Einfallspunktes) ist,

IV. $N=\varrho_{1} \cos e_{1}^{2}$.

Für die Brechung dagegen ist der Aplanatismus abhängig von $x_{0}$ und $e_{2}$; also gilt dieselbe Gleichung für die Fläche in ihrer ganzen Ausdehnung nur für $x_{0}=\infty$. Es lässt sich nun zeigen, dass die Gleichung IV eine Eigenschaft aller Curven zweiter Ordnung ist. Wir gehen aus won der allgemeinen Polargleichung

$$
r=\frac{p}{1+e \cos \vartheta} \text { oder }-\frac{1}{r}=\frac{e}{p} \cos \vartheta+\frac{1}{p} .
$$


Die Differenzirung ergibt

und die Logarithmirung dieser Relation

$$
\frac{d r}{d \vartheta}=\frac{r^{2} e}{p} \sin \vartheta
$$

$$
\lg \frac{d r}{d y}-2 \lg r-\lg \sin \vartheta-\lg \frac{e}{p}=0 .
$$

Differenziren wir abermals, so resultirt

$$
\text { v. } \frac{d^{2} r}{d \vartheta^{2}}-\frac{2}{r}\left(\frac{d r}{d \vartheta}\right)^{2}-\cos \vartheta \frac{d r}{d \vartheta}=0 .
$$

Nun ist die Normale

und weiter

$$
N=\frac{r \sin \vartheta}{\sin \left(\vartheta-e_{1}\right)}=\frac{r \sin \vartheta}{\sin \vartheta \cos e_{1}-\cos \vartheta \sin e_{1}}
$$

woraus folgt

$$
\tan e_{1}=\frac{d r}{r d \vartheta}
$$

$$
\cos e_{1}=\frac{r d \vartheta}{\sqrt{d r^{2}+r^{2} d \vartheta^{2}}}, \sin e_{1}=\frac{d r}{\sqrt{d r^{2}+r^{2} d \vartheta^{2}}} .
$$

Der Werth der Normale verwandelt sich dadurch in

$$
N=\frac{r \sqrt{d r^{2}+r^{2} d \vartheta^{2}}}{r d \vartheta-\cot \vartheta d r} \text {. }
$$

Ferner ist in Berücksichtigung der Differenzialgleichung des Kegelschnitts (V)

$$
\begin{aligned}
& \varrho_{1}= \frac{\left(d r^{2}+r^{2} d \vartheta^{2}\right)^{3 / 2}}{2 d r^{2} d \vartheta-r d^{2} r d \vartheta+r^{2} d \vartheta^{3}}=\frac{\left(d r^{2}+r^{2} d \vartheta^{2}\right)^{3 / 2}: r d \vartheta^{3}}{\frac{2 d r^{2}}{r d \vartheta^{2}}-\frac{d^{2} r}{d \vartheta^{2}}+r} \\
&=\frac{\left(d r^{2}+r^{2} d \vartheta^{2}\right)^{3 / 2}}{r d \vartheta^{2}(r d \vartheta-\cot \vartheta d r)}=\frac{N\left(d r^{2}+r^{2} d \vartheta^{2}\right)}{r^{2} d \vartheta^{2}} .
\end{aligned}
$$

Mithin ist, wie in IV gefordert wurde,

$$
N=\varrho_{1} \cos e_{1}^{2} \text { (Newton). }
$$

Beispielsweise ist für das Ellipsoid $\varrho_{1}=a^{2} N^{3}: b^{4}, \cos e_{1}=$ $b^{2}: a N$; für das gleichseitige Hyperboloid $\varrho_{1}=N^{3}: a^{2}, \cos e_{1}=a: N$. Es lässt sich nun auch in umgekehrter Reihenfolge das 3 . Theorem beweisen. Bezüglich des noch unbestimmten Convergenzpunktes aller gebrochenen und gespiegelten Strahlen und einer beliebigen durch ihn gelegten Achse möge $f(r, 9)=0$ die Gleichung der Achsenschnittcurve einer der Flächen von der vorausgesetzten Eigenschaft sein. Es ist die Function $f(r, \vartheta)$ zu bestimmen aus der Eigensehaft, dass der Winkel $e_{1}$, welcher sich für einen beliebigen Curvenpunkt aus der Gleichung IV ergibt, auch der Winkel zwischen der Normale $N$

E. Pflüger, Archiv für Physiologie. Bd. 91. 
und dem rad. vect. $r$ ist. $N, \varrho_{1}$ und $\cos e_{1}$ lassen sich dureh $r, \vartheta$ und ihre Differenziale ausdrücken. Man hat

folglich

$$
\tan e_{1}=\frac{d r}{r d y}
$$

$$
\begin{aligned}
& \cos e_{1}=\frac{r d \vartheta}{\sqrt{d r^{2}+r^{2} d \vartheta^{2}}}, \sin e_{1}=\frac{d r}{\sqrt{d r^{2}+r^{2} d \vartheta^{2}}}, \\
& N=\frac{r \sqrt{d r^{2}+r^{2} d \vartheta^{2}}}{r d \vartheta-\cot \vartheta d r}, \varrho_{1}=\frac{\left(d r^{2}+r^{2} d \vartheta^{2}\right)^{3 / 2}}{r d \vartheta^{3}\left(\frac{2 d r^{2}}{r d \vartheta^{2}}-\frac{d^{2} r}{d \vartheta^{2}}+r\right)} .
\end{aligned}
$$

Setzen wir diese Werthe in die Gleichung IV ein, so ergibt sich V:

$$
\frac{d^{2} r}{d \vartheta^{2}}-\frac{2}{r} \cdot \frac{d r^{2}}{d \vartheta^{2}}-\cot \vartheta \cdot \frac{d r}{d \vartheta}=0
$$

Die Integration dieser Gleichung führt zur Achsenschnittcurve der gesuchten Fläche. Man bringt sie auf die Form

$$
\frac{d\left(\frac{d r}{d \bar{\vartheta}}\right)}{\frac{d r}{d \boldsymbol{v}}}-2 \frac{d r}{r}-\cot \vartheta d \vartheta=0 .
$$

Die Integration ergibt, wenn $\lg C_{1}$ eine willkürliche Constante bezeichnet,

oder

$$
\lg \frac{d r}{d y}-2 \lg r-\lg \sin \vartheta-\lg C_{1}=0,
$$

$$
\frac{d \boldsymbol{r}}{\boldsymbol{r}^{2}}=C_{1} \sin \vartheta d \boldsymbol{\theta}
$$

Eine weitere Integration ergibt, wenn $C_{2}$ eine andere willkürliche Constante ist

oder

$$
\frac{1}{r}=C_{1} \cos \vartheta+C_{2}
$$

$$
r=\frac{\frac{1}{C_{2}}}{1+\frac{C_{1}}{C_{2}} \cos \vartheta}=\frac{p}{1+e \cos \vartheta} \text { (Detels) }
$$

diese Gleichung ist aber die allgemeine Polargleichung der drei Kegelschnitte. Der Pol ist der verlangte Convergenzpunkt sämmtlicher Strahlen, und die Achse des Polar-Coordinatensystems ist die grosse Achse der Kegelschnitte. 
Wir wollen nun noch ein Mal die Bedingungsgleichung des Aplanatismus für den Fall der Brechung betrachten, indem wir eine Rotationsfläche voraussetzen. Es ist dann nach III a

$$
\text { VI. } x_{0}\left(N-\varrho_{1} \cos e_{1}^{2}\right)=\sin e_{1} \sin \left(e_{2}+e_{1}\right) \varrho_{1} N \text {. }
$$

Wir fanden, dass für vollkommenen Aplanatismus $x_{0}=\infty$, also der leuchtende Punkt unendlich entfernt sein müsse. Man wird indessen noch immer für gewisse Fälle eine genügende Correction des Astigmatismus erwarten dürfen, wenn $x_{0}$ sehr gross gegen die Werthe von $\varrho_{1}$ und $N$ bleibt. In einem solchen Falle würde, da $x_{0}$ wesentlich negativ ist, bei positiven $\varrho_{1}$ und $N$ (Ellipsoid), der Ausdruck $N-\varrho_{1} \cos e_{1}{ }^{2}$ auch negativ sein müssen, also etwa die Bedingungsg]eichung lauten

$$
x_{0}\left[N-\varrho_{1} \cos \left(e_{1}-\Delta e_{1}\right)^{2}\right]=\sin e_{1} \sin \left(e_{2}+e_{1}\right) \varrho_{1} N .
$$

Alsdann würde der erforderliche Strahl nicht genau durch den Focus gehen, aber doch in unmittelbarer Nähe die Achse schneiden. Entwickeln wir diese Gleichung mit Vernachlässigung sehr kleiner Grössen zweiter Ordnung, so resultirt die Anomalie

$$
\text { VII. } \Delta e_{1}=-\frac{\sin \left(e_{2}+e_{1}\right) N}{2 x_{0} \cos e_{1}} \text {. }
$$

Diese Gleichung der Anomalie im Vereine mit dem 1. und 2. Theoreme lassen eine interessante Anwendung auf einen concreten Fall zu, nämlich auf die Theorie der Refraction der ellipsoidischen Gestalt der Hornhaut des menschlichen Auges bei Periscopie. Die Constitution der Hornhaut und die Lage der Pupille und der Linse bringt es mit sich, dass alle peripherischen Strahlen im seitlichen Gesichtsfelde nahezu durch die vordere Hälfte der Linsenachse, also durch einen festen Punkt gehen, und dass der erste Focus des Hornhautellipsoids in der vorderen Hälfte der Linsenachse liegt. Dies gilt auch, soweit Messungen vorliegen, für die Augen der Wirbelthiere überhaupt, wenn der Augapfel ein Rotationskörper ist ${ }^{1}$ ). Wir wollen dies Problem etwas eingehender verfolgen, indem dabei die Gleichungen IV und VI zur Anwendung kommen.

Die ersten zuverlässigen Messungen der Krümmungsverhältnisse der Hornhaut sind von Senff, v. Helmholtz und seinen Schülern ausgeführt, letztere mit Anwendung des Ophthalmometers. Helm-

1) L. Matthiessen, Festschrift zum 70. Geburtstag von Herm. v. Helmholtz. 1891. Tab. 4. 
holtz ging bereits 1854 nach dem Vorgange von Senff von der Annahme aus, dass die Form der Hornhaut nahezu einem oblongen Rotationsellipsoide entspreche. Der Scheitel desselben entspricht äusserlich, wie die Messungen erweisen, nahezu der Mitte der Hornhaut. Unter der erwähnten Voraussetzung maass Helmholtz die Krümmungsradien in der Gesichtslinie und durch Drehung der Gesichtslinie des untersuchten Auges auch die Krümmungsradien zweier anderer seitlich gelegenen Fixationspunkte der Hornhaut. Aus diesen drei Werthen lassen sich die Achsenverhältnisse und die Lage der grossen Achse des hypothetischen Ellipsoids gegen die Gesichtslinie berechnen ${ }^{1}$ ). Später ist noch eine grosse Anzahl von Messungen der Krümmungen und Dimensionen der Hornhaut mit dem Ophthalmometer gemacht worden, besonders von H. A ubert ${ }^{2}$ ). Um den Einwänden 'gegen die Senff-Helmboltz'sche Annahme einer ellipsoidischen Krümmung zu begegnen, da ja doch ohne dieselbe die Messung von nur drei Krümmungsradien nicht auch das Vorhandensein irgend einer anderen Fläche ausschliesst, wurde von $\mathrm{Aubert}$ eine grössere Zahl (7-9) Fixationspunkte an demselben Auge gewählt und die Constanten unter der gleichen Annahme zu Mittelwerthen combinirt. Aber trotz der Jahre langen eifrigen und mit grösstmöglicher Genauigkeit mit Hülfe seines von ihm verbesserten Ophthalmometers gemachten Messungen rerzweifelte er scheinbar schliesslich an der Möglichkeit, durch die Helmholtz'sche Methode die Hornhautober- fläche festzustellen. Er machte versehiedene Vorschläge zur Verbesserung der Methode, welche der berühmte Physiologe nicht mehr zur Ausführung bringen konnte. Aus diesem Grunde haben andere Ophtalmologen zu anderen, völlig davon verschiedenen Methoden gegriffen.

Prof. Allvar Gullstrand in Stockholm ${ }^{8}$ ) hat ohne Ophthalmometer ganz unabhängig von der Hypothese eines Hornhautellipsoides durch eine photographisch-optische Methode eine grosse Anzahl von

1) H. Helmholtz, Gräfe's Arch. f. Ophthalmologie Bd. 1 (2). 1854. Physiologische Optik 1. Aufl. 1866.

2) F. C. Donders in Gräfe's Arch. f. Ophthalm. Bd. 8 (1). 1862 H. A ubert in Pflüger's Arch. f. d. ges. Physiol. Bd. 35 S. 610. 1885, Bd. 49 S. $629,636.1891$.

3) A. Gullstrand, Photographisch-o, ,hthalmometrische und klinische Untersuchungen über die Hornhautrefraction. Kongl. Svenska Vetenskabs-Academiens Handlingar Bd. 28 Nr. 7. 1896. 
Fixationspunkten gemessen und daraus zur Reconstruction der Hornhautfläche in zwei auf einander senkrechten Meridianen eine Tabelle in rechtwinkligen Coordinaten zur Hornhautachse berechnet, und zwar in jedem der beiden Meridiane mit 18 Coordinaten. Mit Hülfe dieser Tabelle sind von mir für den horizontalen Meridian unter Anwendung der hypothetischen Ellipsengleichung

$$
y^{2}=2 \frac{b^{2}}{a} x-\frac{b^{2}}{a^{2}} x^{2}=2 \varrho_{0} x-\frac{b^{2}}{a^{2}} x^{2}
$$

alle tangirenden Ellipsen berechnet, und zwar so, dass immer zusammengehörige Coordinatenpaare mit dem bereits ziemlich sicher bestimmten Krümmungsradius $\varrho_{0}$ des Scheitels in diese Formel eingesetzt und daraus die zugehörigen Halbachsen $a$ und $b$ sowie der Abstand $a-\sqrt{a^{2}-b^{2}}$ des Focus vom Scheitel gesucht wurden. Betrachtet man aber zuvor noch $\varrho_{0}$ als unbekannt, so lässt sich zunächst aus möglichst vielen Combinationen nicht nahe zusammenliegender Coordinatenpaare durch Einführung in die Ellipsengleichung ein guter Mittelwerth $\varrho_{0}$ berechnen, wofür im Mittel $7,66 \mathrm{~mm}$ gefunden wurde. Derselbe zeigt mit dem des Auges $V$ von $K n$ app $7,81 \mathrm{~mm}$ und Helmboltz $7,71 \mathrm{~mm}$ eine genügende Uebereinstimmung. Aus der Gleichung

$$
y^{2}=2 \cdot 7,66 x-\frac{b^{2}}{a^{2}} x^{2}
$$

ergeben sich somit die Einzelwerthe der tangirenden Ellipsen $a, b$ und $a-\sqrt{a^{2}-b^{2}}$, wie folgende Tabelle zeigt. (Man vergl. Gullstrand Tab. 17).

\begin{tabular}{|c|c|c|c|c|c|c|}
\hline Punkt & $x \mathrm{~mm}$ & $y \mathrm{~mm}$ & $\varrho_{0}=\frac{b^{2}}{a}$ & $a \mathrm{~mm}$ & $b \mathrm{~mm}$ & $a-\sqrt{a^{2}-b^{2}}$ \\
\hline $\begin{array}{l}1 \\
2 \\
3 \\
4 \\
5 \\
6 \\
7 \\
8 \\
9\end{array}$ & $\begin{array}{l}0,00675 \\
0,06137 \\
0,17266 \\
0,34574 \\
0,57264 \\
0,86002 \\
1,23058 \\
1,79222 \\
2,44005\end{array}$ & $\begin{array}{l}0,32127 \\
0,96831 \\
1,62034 \\
2,28387 \\
2,92273 \\
3,55572 \\
4,21771 \\
5,04841 \\
5,85055\end{array}$ & $\begin{array}{l}7,66 \\
7,66 \\
7,66 \\
7,66 \\
7,66 \\
7,66 \\
7,66 \\
7,66 \\
7,66\end{array}$ & $\begin{array}{l}\overline{11,22} \\
11,62 \\
11,35 \\
10,90 \\
10,64 \\
10,91 \\
12,49 \\
14,46\end{array}$ & $\begin{array}{r}\overline{9,27} \\
9,43 \\
9,32 \\
9,14 \\
9,03 \\
9,14 \\
9,78 \\
10,53\end{array}$ & $\begin{array}{l}\overline{4,90} \\
4,83 \\
4,87 \\
4,96 \\
5,01 \\
4,95 \\
4,72 \\
4,54\end{array}$ \\
\hline & & & Mittel & 11,699 & 9,455 & 4,848 \\
\hline
\end{tabular}

N a s a l. 
Temporal.

\begin{tabular}{|c|c|c|c|c|c|c|}
\hline Punkt & $x \mathrm{~mm}$ & $y \mathrm{~mm}$ & $\varrho_{0}=\frac{l^{2}}{a}$ & $a \mathrm{~mm}$ & $b \mathrm{~mm}$ & $a=\sqrt{a^{2}-b^{2}}$ \\
\hline $\begin{array}{l}1 \\
2 \\
3 \\
4 \\
5 \\
6 \\
7 \\
8 \\
9\end{array}$ & $\begin{array}{l}0,00675 \\
0,06187 \\
0,17231 \\
0,34030 \\
0,56176 \\
0,83146 \\
1,16784 \\
1,57868 \\
2,13860\end{array}$ & $\begin{array}{l}0,32127 \\
0,97431 \\
1,62135 \\
2,26538 \\
2,88892 \\
3,48311 \\
4,08405 \\
4,69169 \\
5,38499\end{array}$ & $\begin{array}{l}7,66 \\
7,66 \\
7,66 \\
7,66 \\
7,66 \\
7,66 \\
7,66 \\
7,66 \\
7,66\end{array}$ & $\begin{array}{c}\overline{20,50)} \\
(-20,68 \\
10,84 \\
9,29 \\
8.74 \\
8,62 \\
8,78 \\
9,31\end{array}$ & $\begin{array}{c}\overline{(12,53} \sqrt{-1}) \\
12,59 \\
9,11 \\
8,43 \\
8,18 \\
8,13 \\
8,20 \\
8,44\end{array}$ & $\begin{array}{c}\overline{(3,53)} \\
4,27 \\
4,97 \\
5,40 \\
5,65 \\
5,74 \\
5,64 \\
5,39\end{array}$ \\
\hline & $15 \mathrm{Me}$ & $\mathrm{Ge}$ & $\begin{array}{l}\text { Mittel } \\
\text { tmittel }\end{array}$ & $\begin{array}{l}10,894 \\
11,296\end{array}$ & $\begin{array}{l}9,011 \\
9,233\end{array}$ & $\begin{array}{l}5,294 \\
5,071\end{array}$ \\
\hline
\end{tabular}

Sieht man vun der ersten Messung an der nasalen Seite und von den beiden ersten Messungen an der temporalen Seite ab, so ergibt sich aus den übrigen 15 Coordinaten eine Grundlage für die Herstellung einer Hornhautellipse im horizontalen Meridian, welche sich den Mittelwerthen der Messungen anderer Autoren recht gut anschliesst, wie folgende Zusammenstellung erweist.

\begin{tabular}{|c|c|c|c|c|c|c|}
\hline Autoren & $\vartheta_{0}$ & $a$ & $b$ & $a: b$ & $a-\sqrt{a^{2}-b^{2}}$ & $\frac{\varepsilon=}{\sqrt{a^{2}-b^{2}}: a}$ \\
\hline $\begin{array}{l}\text { Senff. } \\
\text { Helmpoitz II-IV } \\
\text { Knapp V-IX. } \\
\text { Knapp hor. u. vert. } \\
\text { Gullstrand. }\end{array}$ & $\begin{array}{l}7,79 \\
7,71 \\
7,61 \\
7,81 \\
7,66\end{array}$ & $\begin{array}{l}10,435 \\
11,613 \\
10,580 \\
11,009 \\
11,296\end{array}$ & $\begin{array}{l}9,019 \\
9,446 \\
8,916 \\
9,272 \\
9,233\end{array}$ & $\begin{array}{l}1,157 \\
1,230 \\
1,186 \\
1,187 \\
1,223\end{array}$ & $\begin{array}{l}5,186 \\
4,858 \\
4,885 \\
5,073 \\
5,071\end{array}$ & $\begin{array}{l}0,503 \\
0,582 \\
0,538 \\
0,539 \\
0,551\end{array}$ \\
\hline
\end{tabular}

Eine andere Methode der Messung möglichst vieler Coordinaten ist von meinen Schülern Mönni $\mathrm{Ch}^{1}$ ), Klingberg ${ }^{2}$ ) und Me yer ${ }^{3}$ ) mit Erfolg angewendet worden. Mönnich stellte sorgfältige negative Gypsabgüsse der Hornhaut von frischen, todten Augen des Rindes her und von diesen mehrere positive, welche er in der Richtung eines der beiden Meridiane fein durchsägte, nachdem der Scheitelpunkt möglichst nahe festgestellt war. Von diesen secundären Schnitten

1) P. Mönnich, Ueber den physikalisch-optischen Bau des Rindsanges. Zeitschr. f. vergl. Augenheilkunde von Berlin u. Fversbusch Bd. 2. 1883.

2) A. Klingberg, Beiträge zur Dioptrik der Augen einiger Hausthiere. Güstrow. Progr. 1888, 1889, 1892.

3) W. Meyer, Ueber den physikalisch-optischen Ban vom Schaf und Hund. Inaug.-Diss. Rostock 1897. 
stellte er sodann mittelst eines achromatischen Skioptikons zehnfach vergrösserte Projectionen und genaue Risse derselben her, welche auf Coordinaten gemessen wurden, nachdem davon die Symmetrieachse der Schmiegungseurve genau festgestellt war. Um jede etwaige Verzerrung durch die Projection zu vermeiden, wurden die Schnitte auf Glasplatten mit feinen Liniensystemen geklebt. Dabei ergaben sich zwei Resultate; erstens, dass die beiden Hauptmeridiane verschiedene Krümmungen, zweitens eine merkliche Ellipticität besitzen. Die Ellipticitäten wurden dann aus den gemessenen Coordinaten bestimmt nach einem ähnlichen Verfahren, welches oben bei den Gullstrandschen Messungen von mir angewendet ist. Die beobachteten Werthe, mit den berechneten verglichen, zeigten eine sehr genaue Uebereinstimmung. Es mögen hier eine Zusammenstellung der Constanten für den horizontalen Meridian folgen.

\begin{tabular}{|c|c|c|c|c|c|c|}
\hline Autoren und Augen & $\varrho_{0}$ & $a$ & $b$ & $a: b$ & $f$ & $\varepsilon$ \\
\hline $\begin{array}{l}\text { Knapp, Mensch . . . } \\
\text { Mönnich, Rind (8. Aug.). } \\
\text { Klingberg, Pferd (4. Aug.). } \\
\text { Klingberg, Schaf (4. Aug.) } \\
\text { Meyer, Schaf (4. Aug.). . } \\
\text { Klingberg, Schwein (5. Aug.) } \\
\text { Klingberg, Katze (5. Aug.). } \\
\text { Meyer, Hund (3. Aug.). }\end{array}$ & $\begin{array}{r}7,81 \\
15,18 \\
17,13 \\
9,99 \\
11,31 \\
8,20 \\
7,33 \\
8,66\end{array}$ & $\begin{array}{l}11,01 \\
29,13 \\
39,66 \\
16,61 \\
17,89 \\
16,04 \\
10,71 \\
16,49\end{array}$ & $\begin{array}{r}9,27 \\
18,52 \\
25,94 \\
12,86 \\
14,22 \\
11,19 \\
8,86 \\
11,90\end{array}$ & $\begin{array}{l}1,187 \\
1,225 \\
1,528 \\
1,282 \\
1,258 \\
1,386 \\
1,209 \\
1,367\end{array}$ & $\begin{array}{l}5,07 \\
9,27 \\
9,66 \\
6,10 \\
7,04 \\
4,55 \\
4,69 \\
5,08\end{array}$ & $\begin{array}{l}0,54 \\
0,60 \\
0,75 \\
0,63 \\
0,61 \\
0,71 \\
0,56 \\
0,69\end{array}$ \\
\hline
\end{tabular}

Aus dieser Uebersicht ergibt sich nun die bereits erwähnte Thatsache, dass bei allen Augen der Wirbelthiere das Centrum des Aplanatismus peripherisch bis zu den äussersten Grenzen des Gesichtsfeldes in das Auge gelangender Strahlen in der vorderen Hälfte der Linse liegt. Bedentet $d_{1}$ den Ort des vorderen Scheitels, $d_{2}$ den des hinteren, also $d_{1}+{ }^{1 / 2} d_{2}$ den Ort der Mitte der Linsenachse, so ergibt sich folgende Zusammenstellung.

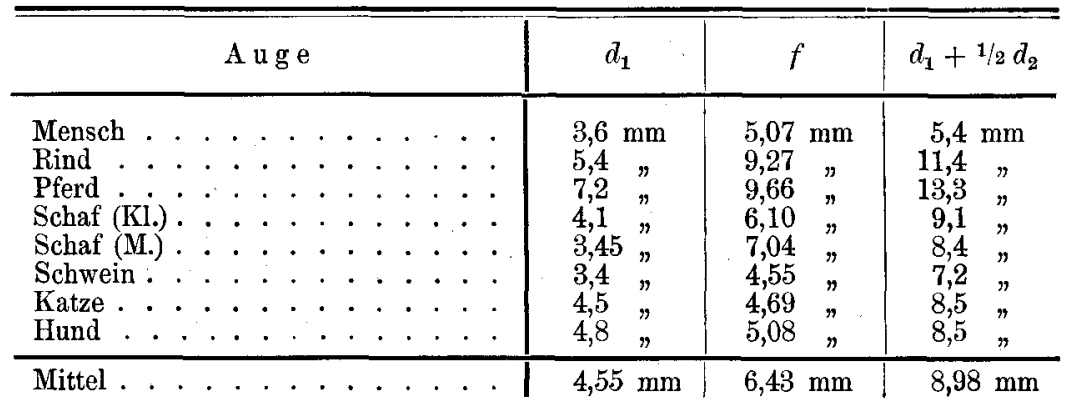


Hieraus geht hervor, dass der Focus der Schmiegungsellipse immer nahezu in der Mitte zwischen Linsenscheitel und Linsenmitte liegt.

Die obigen Ergebnisse drängen uns nun folgende Fragen auf:

1. Ist die unzweifelhaft vorhandene elliptische Krümmung eine blosse Wirkung des intraocularen Druckes ohne eine optische Bestimmung, oder findét eine Beziehung zur letzteren statt?

2. Ist sie bestimmt oder geeignet für die Gorrection eines Astigmatismus der in der Gesichtslinie eintretenden Strahlen mit diesem verhältnissmässig kleinen Gesichtsfelde?

3. Welche Kegelsehnitte sind allein geeignet für den Aplanatismus der Hornhaut?

4. Uebt die ellipsodische Gestalt eine aplanatische Wirkung auf das ganze übrige verhältnissmässig grosse seitliche Gesichtsfeld aus?

Die erste Frage kann wohl im Ganzen dahin beantwortet werden, dass der intraoculare Druck nothwendig ist für die Glättung und Straffheit der Hornhaut. Wenn das Hornhautellipsoid eine Catenoidalfläche wäre, so könnte diese je nach der Constitution der Haut eine sehr verschiedene Form haben.

Die zweite Frage lässt sich mathematisch beantworten. Bei einem vom Scheitel nach der Peripherie sich immer mehr abflachenden Ellipsoide liegt die Normale hinter dem vorderen Brennpunkte. Eine aplanatische Wirkung desselben ist nur dann möglich, wenn die einfallenden, von einem verhältnissmässig weit entfernten paraxialen Objecte herkommenden Strahlen nach der Brechung in der Hornhaut gegen den hinteren Brennpunkt der Ellipsoides gerichtet sind. Nun ist die zweite Hauptbrennweite des Scheitelflächenelementes

$$
\frac{n \varrho_{0}}{n-1}=a+\sqrt{a^{2}-b^{2}}
$$

oder durch die Excentricität ausgedrückt

$$
\frac{n}{n-1}\left(1-\varepsilon^{2}\right)=1+\varepsilon
$$

folglich müsste der Brechungsindex der Hornhaut mit dem Kammerwasser $n=1: \varepsilon$ betragen. Dies ist aber auch nicht einmal annähernd weder für das menschliche Auge noch für alle anderen bis jetzt gemessenen Thieraugen der Fall. Denn $n$ hat stets nahezu den Werth 1,3365 und $1: \varepsilon$ für das menschliche Auge 1,852 und für die acht verschiedenen Augenarten im Mittel den Werth 1:0,636= 
1,572. Für den Strahlengang im directen Gesichtsfelde hat die Ellipticität absolut keine Bedeutung, ist auch gar nicht erforderlich, wenn nur das senkrecht zur Gesichtslinie gerichtete, sehr kleine Flächenelement sphärisch ist, und sphärisch ist es immer bei allen Kegelschnitten.

Was weiter die dritte Frage betrifft, so ist die Excentricität $\varepsilon$ für die Ellipse +, für den Kreis 0 , für die Parabel 1, für die $\mathrm{Hy}-$ perbel - Da aber. $n$ eine positive Grösse ist, nämlich 1,3365 , so können es ebensowenig die übrigen Kegelschnitte sein. Da $n$ bei festen und flüssigen Medien zwischen 1,333 und 2 schwankt, so würde $\varepsilon$ zwischen 0,75 und 0,5 schwanken, was bei den Augen thatsächlich der Fall ist. Im Uebrigen hat diese Thatsache keine Wichtigkeit für das centrale Gesichtsfeld. Die Brennweite ist nicbt gleich der Focaldistanz $a(1 \pm \varepsilon)$, sondern $n \varrho_{0}:(n-1)$, welche allein der Lage der Retina nach der Brechung in der Linse adaptirt ist. Bei dem menschlichen Auge ist die hintere Hauptbrennweite der Hornhaut $31,2 \mathrm{~mm}$, die zweite Focaldistanz der ellipsoidischen Hornhaut dagegen nur $17,0 \mathrm{~mm}$.

Die vierte Frage ist bereits durch das vorangestellte 3. Theorem erledigt auf Grund der Thatsache, dass alle Strahlen, welche aus dem verhältnissmässig grossen seitlichen Gesichtsfelde, welches bis zu $90^{\circ}$ temporal reicht, noch in die Linse gelangen, nahezu durch einen festen Punkt gehen, welcher in der Mitte der vorderen Achsenhälfte der Linse liegt.

Wir wollen nun noch prüfen, welche Schwankungen dieses als fest angenommenen aplanatischen Centrums noch gestattet sind, um der Bedingungsgleichung

zu genügen.

$$
\text { IV } N=\varrho, \cos e_{1}^{2}
$$

Es möge dies an einem concreten Falle, dem menschlichen Auge, erläutert werden, indem wir die Krümmungsverhältnisse von Knapp $\mathrm{V}$ zu Grunde legen, nämlich

$$
\begin{array}{ccc}
\varrho_{0} & a & b \\
7,81 \mathrm{~mm} & 11,01 \mathrm{~mm} & 9,27 \mathrm{~mm} .
\end{array}
$$

Bezeichnen wir den Winkel, welchen die Normale des Incidenzpunktes mit der optischen Achse bildet, mit $\omega$, die Ordinate mit $y$ den Winkel, welchen der rad. vect. $r$ mit der Achse bildet, mit $\lambda$ und den halben Winkel des Sehfeldes mit $\mu$. Nun ist von Aubert für die äusserste noch messbare Grenze der Hornhaut $\omega=27^{\circ} 45^{\prime}$, 
von Gullstrand $y=5,4 \mathrm{~mm}$ gefunden. Wir gehen aus von der Ordinate $g=4,5 \mathrm{~mm}$. Dann gelten folgende Formeln

$$
\begin{gathered}
\varrho_{0}=\frac{b^{2}}{a}, \varrho=\frac{\left[b^{4}+y^{2}\left(a^{2}-b^{2}\right)\right]^{3 / 2}}{a b^{4}}, \\
N^{3}=\frac{b^{4}}{a^{2}} \varrho, \cos e_{1}=\frac{b^{2}}{a N}, \lambda=\frac{\pi}{2}-\left(\arccos \frac{y}{N}-\arccos \frac{\varrho_{0}}{N}\right) \\
r=y: \sin \lambda, \omega=\lambda-e_{1}, \mu=\lambda+e_{2}-e_{1} .
\end{gathered}
$$

Die Berechnung ergibt folgende Zahlenwerthe: $y=4,5 \mathrm{~mm}, \varrho=8,981, N=8,181, e_{1}=17^{\circ} 16^{\prime} 30^{\prime \prime}, e_{2}=23^{\circ} 23^{\prime} 0^{\prime \prime}$, $\lambda=50^{\circ} 38^{\prime} 30^{\prime \prime}, \omega=33^{\circ} 22^{\prime} 0^{\prime \prime} \mu=56^{\circ} 35^{\prime} 0^{\prime \prime}, r=5,820$.

Wir prüfen hiermit die Anomalie

$$
\text { VII. } \Delta e_{1}=-\frac{\sin \left(e_{2}+\epsilon_{1}\right) N}{2 x_{0} \cos e_{1}} \text {, }
$$

und zwar für einen ziemlich ungünstigen Fall, nämlich $x_{0}=-$ $250 \mathrm{~mm}$, die Entfernung des deutlichen Sehens, statt $x_{0}=\infty$. Wir finden $\Delta e_{1}=+0^{0} 38^{\prime} 25^{\prime \prime}$ und die Verschiebung des aplanatischen Centrums auf der Linsenachse

$$
z=\frac{r \sin \Delta e_{1}}{\sin \left(\lambda-\Delta e_{1}\right)}=\frac{5,820 \cdot \sin 0^{\circ} 38^{\prime} 25^{\prime \prime}}{\sin 50^{\circ} 0^{\prime} 30^{\prime \prime}}=0,085 \mathrm{~mm} .
$$

Die Verschiebung des aplanatischen Centrums würde noch nicht einmal $0,1 \mathrm{~mm}$ betragen.

Was nun die allgemeine Bedingungsgleichung des Aplanatismus, nämlich

$$
\text { IIIa. } x_{0}\left(\varrho_{2}-\varrho_{1} \cos e_{1}^{2}\right)=\sin e_{1} \sin \left(e_{2}+e_{1}\right) \varrho_{1} \varrho_{2}
$$

anbetrifft, so geht aus ihr hervor, dass auch bei der Brechung für eine endliche Objectsphäre von der Distanz $x_{0}$ dieselbe den Astigmatismus peripherischer Strahlenbündel corrigiren kann, wenn $\varrho_{2}$ von $N$ verschieden ist, es sich also nicht um eine Rotationsfläche handelt. Da $x_{0}$ für divergente Strahlen (reelle Objecte) negativ ist, so muss $\varrho_{2}$ einen kleineren Werth als $N$ haben und, da $x_{0}$ für convergente Strahlen (imaginäre Objecte) positiv ist, so muss $\varrho_{2}$ einen grösseren Werth als $N$ haben. Daraus geht hervor, dass auch eine dreiachsige Fläche II. Ordnung für peripherische Strahlen aplanatisch werden kann. Nehmen wir an, es sei dies ein dreiachsiges Ellipsoid, welches freilich auf Augen nicht völlig anwendbar zu sein scheint, da die grosse Hornhautachse $2 a$ wohl niemals den beiden Hauptmeridianen gemeinschaftlich ist. Deswegen bin ich auch nicht geneigt, der hypothetischen Kugelzone im Hornhautscheitel (A ubert) zuzustimmen, worauf ich weiter unten zurückkommen werde. Man wird 
aber zugestehen müssen, dass es sehr wohl möglich ist, dass die beiden Hauptmeridiane eine vollkommene elliptische Krümmung haben, ohne dass die ganze Hornhautfläche ein dreiachsiges Ellipsoid ist. Indessen gehen wir von einem concreten Falle aus, der den Krümmungsverhältnissen der menschlichen Hornhaut sich nähert. Kn a p p fand in 16 Fällen den horizontalen Meridian schwächer gekrümmt als den verticalen. So ist es immer der Fall bei Thieren, welche eine längsovale Pupille besitzen, wie bei den Hufthieren und den Walfischen. Wir sehen dabei ab von dem Winkel $\alpha$, welchen die Gesichtslinie mit der grossen Achse des dreiachsigen Ellipsoides bildet. Es seien für den

$$
\begin{aligned}
\text { horizontalen Meridian } a=11,01 \mathrm{~mm}, b=9,27 \mathrm{~mm}, \\
\text { verticalen } \quad a=11,01 \quad \Rightarrow b=9,00 \%
\end{aligned}
$$

also $a>b>c$. Für divergente Strahlen $\left(x_{0}-\right)$ ist alsdann für einen Incidenzpunkt im horizontalen Meridian $\varrho_{2, h}<N_{h}$, im verticalen $\varrho_{2, v}>N_{v}$. Wir suchen die Werthe von $\varrho, \varrho_{1}, \varrho_{2}$ in beiden Meridianen, unter der Voraussetzung, dass in beiden Fällen der einfallende, unendlich dünne Strahlenfächer in der Meridianebene liegt, und dass der gebrochene Fächer durch den Focus oder mindestens durch die vordere Linsenhälfte geht. Es ist

$$
\frac{x^{2}}{a^{2}}+\frac{y^{2}}{b^{2}}+\frac{z^{2}}{c^{2}}=1 \text {, }
$$

für den horizontalen Meridian $(a, b)$

$$
\begin{gathered}
N_{h}=y \sqrt{1+\left(\frac{d y}{d x}\right)^{2}}=\frac{1}{a^{2}} \sqrt{a^{4} y^{2}+b^{4} x^{2}}=\frac{1}{a} \sqrt{b^{4}+y^{2}\left(a^{2}-b^{2}\right)}, \\
\varrho_{0, h}=\frac{b^{2}}{a}, \varrho_{1, h}==\frac{a^{2}}{b^{4}} N_{h}^{3}, \varrho_{2, h}=\frac{c^{2}}{b^{2}} N_{h}, \cos e_{1}=\frac{b^{2}}{a N} \\
\sin e_{2}=1,3365 \sin e_{1}, f_{h}=a-\sqrt{a^{2}-b^{2}}
\end{gathered}
$$

für den verticalen Meridian $(a, c)$

$$
\begin{gathered}
N_{v}=\frac{1}{a} \sqrt{c^{4}+y^{2}\left(a^{2}-c^{2}\right)}, \varrho_{0, v}=\frac{c^{2}}{a}, \varrho_{1, v}=\frac{a^{2}}{c^{4}} N_{v}{ }^{3}, \varrho_{2, v}=\frac{b^{2}}{c^{2}} N_{v}, \\
\quad \cos e_{1}=\frac{c^{2}}{a N}, \sin e_{2}=1,3365 \sin e_{1}, f_{v}=a-\sqrt{a^{2}-c^{2}} .
\end{gathered}
$$

Wir berechnen diese Werthe aus den gegebenen $y=4,5 \mathrm{~mm}$, $a, b$ und $c$. Wir finden im horizontalen Meridian:

$$
\begin{gathered}
N_{h}=8,131, \varrho_{0, h}=7,805, \quad \varrho_{1, h}=8,981, \varrho_{2, h}=7,705, \varrho_{2, h}<N_{h}, \\
e_{\mathbf{1}}=17^{\circ} 16^{\prime} 30^{\prime \prime}, \quad e_{2}=23^{0} 23^{\prime} 0^{\prime \prime}, \quad f=a-\sqrt{a^{2}-b^{2}}=5,07 ;
\end{gathered}
$$

im verticalen Meridian: 


$$
\begin{gathered}
N_{v}=7,800, \varrho_{0, v}=7,357, \quad \varrho_{1, v}=8,768, \quad \varrho_{2, v}=8,275, \varrho_{2, v}>N_{\nu}, \\
e_{1}=19^{\circ} 24^{\prime} 0^{\prime \prime}, \quad e_{2}=26^{\circ} 21^{\prime} 30^{\prime \prime}, \quad f=a-\sqrt{a^{2}-c^{2}}=4,67 .
\end{gathered}
$$

Die beiden Brennpunkte liegen demnach in der vorderen Linsenhälfte. Wir setzen die vorstehenden Zahlenwerthe in die aplanatischen Gleichungen ein,

III a. $x_{0}\left(\varrho_{2}-\varrho_{1} \cos e_{1}^{2}\right)=\sin e_{1} \sin \left(e_{2}+e_{1}\right) \varrho_{1} \varrho_{2}$,

III b. $x_{2}\left(\varrho_{2}-\varrho_{1} \cos e_{2}^{2}\right)=\sin e_{2} \sin \left(e_{2}+e_{1}\right) \varrho_{1} \varrho_{2}$.

Für den horizontalen Meridian ist also $x_{v}$ negativ (reelle Objecte, reelle Bilder); und zwar

$$
x_{0}=-27,67 \mathrm{~mm}, x_{2}=+129,33 \mathrm{~mm} \text {; }
$$

für den verticalen Meridian ist $x_{0}$ positiv (imaginäre Objecte, reelle Bilder);

$$
x_{0}=+36,43 \mathrm{~mm}, x_{2}=+18,66 \mathrm{~mm} \text {. }
$$

Hieraus folgt nunmehr das

4. Theorem. Die beiden Hauptmeridiane eines dreiachsigen Ellipsoides sind aplanatische Curven für Strahlenfächer in jenen Ebenen, und zwar der schwächer gekrümmte für bestimmte endlich entfernte peripherische monoculare Horopter ausserhalb des Ellipsoides, der stärker gekrümmte für endlich entfernte peripherische Horopter innerhalb des Ellipsoides.

Da die Gleichung IIIa für beliebige Incidenzpunkte zwei Variabele $x_{0}$ und $e_{1}$ hat, so gibt es für jeden Incidenzwinkel $e_{2}$ immer conjugirte Punkte, für welche Aplanatismus stattfindet. Es ist dabei nach IIIa und IIIb

$$
\begin{aligned}
x_{0} & =\frac{\sin e_{1} \sin \left(e_{2}+e_{1}\right) \varrho_{1} \varrho_{2}}{\varrho_{2}-\varrho_{1} \cos e_{1}{ }^{2}}, \\
x_{1}=x_{2} & =\frac{\sin e_{2} \sin \left(e_{2}+e_{1}\right) \varrho_{1} \varrho_{2}}{\varrho_{2}-\varrho_{1} \cos e_{2}{ }^{2}} .
\end{aligned}
$$

Das 4. Theorem lässt eine Verallgemeinerung zu auf jede beliebige krumme Oberfläche eines brechenden Mediums. Für die Kugel war dies längst bekannt ${ }^{1}$ ). Setzt man nämlich $\varrho_{1}=\varrho_{2}=r$, so werden die aplanatischen Gleichungen

$$
x_{0}=\frac{r \sin \left(e_{2}+e_{1}\right)}{\sin e_{1}}, x_{1}=x_{2}=\frac{r \sin \left(e_{2}+e_{1}\right)}{\sin e_{2}}, x_{0}=n x_{1} .
$$

$x_{0}$ und $x_{1}$ sind also positiv (imaginäre Objecte, reelle Bilder). Die geometrischen Oerter dieser conjugirten Punkte sind zwei mit der

1) Beiträge etc. in Zeitschr. f. vergl. Augenheilkunde Bd. 6 Cap. II $\S 38$. 
Ueber aplanatische Brechung und Spiegelung in Oberflächen etc. $\quad 309$

Kugel concentrische feste Kreise mit den Radien $r n$ und $r: n$ (Weierstrass). Für die beiden Meridiane des dreiachsigen Ellipsoides gibt es je zwei geometrische Oerter, von denen bei dem schwächer gekrümmten $x_{0}$ negativ, $x_{2}$ positiv, bei dem stärker gekrümmten $x_{0}$ positiv, $x_{2}$ positiv ist.

Schliesslich möchte ich noch der späteren kaum gerechtfertigten Annahme Aubert's entgegentreten, der nämlich, dass der Scheitel der menschlichen Hornhaut eine sphärische Zone (Kugelcalotte) sei, und welche von Ophthalmologen weiter getragen ist, ohne mit dem Helmboltz'schen Ophthalmometer selbst gemessen zu haben. Jene Annahme hat ihren Grund in Folgendem. Es ist z. B. für den verticalen Meridian

$$
\varrho_{1}=\frac{c_{2}}{a} \sqrt{1+y^{2} \frac{\left(a^{2}-c^{2}\right)^{3}}{c^{4}}} .
$$

Für kleine, aber keineswegs verschwindende Ordinaten, z. B. $y=2 \mathrm{~mm}$, kann man setzen

$$
\varrho_{1}=\frac{c^{2}}{a}\left(1+{ }^{3 / 2} y^{2} \frac{a^{2}-c^{2}}{c^{4}}\right) .
$$

Man findet $\varrho_{0}=7,36, \varrho_{1}=(7,36+0,27 \mathrm{~mm})$. Also wenn die persönlichen oder Beobachtungsfehler 0 bis $0,27 \mathrm{~mm}$ betragen, was nicht ausgeschlossen ist, so kann sich offenbar leicht obige Ansicht bilden, dass in dem Intervall $y=0$ bis $2 \mathrm{~mm} \varrho_{1}=\varrho_{0}$ zu setzen sei. Um die Annahme Aubert's zu rechtfertigen, müssten in der Nähe des Scheitels der Ellipse viel genauere Messungen angestellt werden. Dies beweisen auch die Ellipticitätstafeln von Gullstrand, der schon für $y=1 \mathrm{~mm}$ die Constanten $\varrho_{0}=7,66, a=11,22, b=9,27$, $f=4,90$ fand. Eine kugelförmige Zone ist offenbar auch nur dann möglich, wenn die Achsenverhältnisse in beiden Meridianen völlig dieselben sind, sonst bleibt die in Rede stehende Zone immer ellipsoidisch, nicht sphärisch.

Es ist nun in der That auffallend, dass die Theoreme 2 und 3 , deren Wichtigkeit für die Dioptrik des Auges von mir bereits im Jahre $1879^{1}$ ) betont ist, in der Opthalmologie bis jetzt keine Beachtung gefunden haben.

1) Pflüger's Arch. f. d. ges. Physiol. Bd. 19 S. 525. 1879. 